\title{
Alimentos ultraprocessados e o consumo de fibras alimentares no Brasil
}

\author{
Ultra-processed foods and dietary fiber consumption in Brazil
}

Gabriela Lopes da Cruz (https://orcid.org/0000-0002-8220-3498) ${ }^{1}$

Priscila Pereira Machado (https://orcid.org/0000-0003-4607-5094) ${ }^{1}$

Giovanna Calixto Andrade (https://orcid.org/0000-0002-1901-3093) ${ }^{1}$

Maria Laura da Costa Louzada (https://orcid.org/0000-0002-3756-2301) ${ }^{1}$

${ }^{1}$ Núcleo de Pesquisas Epidemiológicas em Nutrição e Saúde,

Universidade de São

Paulo. Av. Dr. Arnaldo 715, Pacaembu. 01246-904 São Paulo SP Brasil.

gabriela.cruz@usp.br

\begin{abstract}
The consumption of dietary fiber in Brazil and its relationship with the intake of ultra -processed foods was evaluated. The analysis used food consumption data, with a 24-hour food record of residents aged $\geq 10$ years $(n=34.003)$ from the 2008-2009 Family Budgets Survey. The food products were divided into groups: in natura or minimally processed ingredients; processed culinary ingredients; processed and ultra-processed ingredients. The contribution of each food group and selected subgroups to the total fiber intake, the relation between quintiles of ultra-processed foods (evaluated by the \% of total energy intake), average dietary fiber content $(\mathrm{g} / 1,000 \mathrm{kcal})$, and the prevalence of inadequate fiber consumption, was estimated. In natura or minimally processed foods revealed significantly higher fiber density than ultra-processed foods and corresponded to the majority percentile contribution of dietary fiber, notably derived from rice and beans. Individuals in the largest quintile of ultra-processed consumption were 1.5 times more likely to ingest inadequate fiber intake. The consumption of ultra-processed foods had a negative impact on fiber intake. Reducing the consumption of these foods can bring benefits to the quality of the Brazilian diet.

Key words Dietary fiber, Food consumption, Dietary surveys, Nutritional epidemiology, Industrialized foods
\end{abstract}

Resumo Foi avaliado o consumo de fibras alimentares no Brasil e sua relação com a ingestão de alimentos ultraprocessados. Foram utilizados dados de consumo alimentar, via registro alimentar de 24 horas, com indivíduos de idade $\geq 10$ anos ( $n=34.003$ ) oriundos da Pesquisa de Orçamentos Familiares 2008-2009. Os alimentos foram divididos em grupos: in natura ou minimamente processados, ingredientes culinários processados, processados e ultraprocessados, estimando-se sua contribuição para o consumo de fibras. Verificouse a relação entre quintos de consumo de ultraprocessados (avaliado pelo \% do total de energia consumida) e teor médio de fibras na dieta (g/1.000kcal), e a prevalência de inadequação no consumo de fibras. Alimentos in natura ou minimamente processados apresentaram densidade de fibras significativamente maior do que os ultraprocessados e corresponderam à majoritária contribuição percentual de fibras na dieta, notavelmente a partir do arroz e feijão. Indivíduos do maior quintil de consumo de ultraprocessados tiveram 1,5 vez mais chance de apresentar ingestão de fibras inadequada. O consumo de ultraprocessados impactou negativamente na ingestão de fibras. Reduzir o consumo desses alimentos pode trazer benefícios à qualidade da dieta brasileira.

Palavras-chave Fibras na dieta, Consumo de alimentos, Inquéritos sobre dietas, Epidemiologia nutricional, Alimentos industrializados 


\section{Introdução}

Fibras alimentares são definidas como uma mistura heterogênea de polissacarídeos e ligninas não degradadas pelas enzimas endógenas humanas ${ }^{1,2}$. A ingestão de alimentos ricos em fibras tem papel protetor para diversas doenças crônicas e distúrbios gastrointestinais ${ }^{1}$. Seu consumo regular e adequado está associado à prevenção de câncer de cólon e reto, doença cardiovascular, hipertensão, acidente vascular cerebral, obesidade e diabetes, assim como perda de peso, diminuição da pressão arterial, redução da resposta glicêmica, do colesterol LDL e total ${ }^{1,3}$. O efeito protetor das fibras independe de fatores como idade, índice de massa corporal, tabagismo e prática de atividade física ${ }^{1}$.

Diante dos seus benefícios, a Organização Mundial da Saúde (OMS) recomenda um consumo de fibras alimentares mínimo de $25 \mathrm{~g} / 2.000$ kcal (ou 12,5 g/1.000 kcal) ${ }^{1}$, valor também adotado pelo Ministério da Saúde para a população brasileira $^{4}$.

Os americanos consomem quantidades insuficientes de fibras, alcançando apenas $40 \%$ da sua recomendação nacional $(14 \mathrm{~g} / 1.000 \mathrm{kcal})^{5}$; o consumo médio da população adulta do Reino Unido equivale a menos de $65 \%$ do recomendado pelo seu guia alimentar $(30 \mathrm{~g} / \mathrm{dia})^{6}$.

Sardinha et al. ${ }^{7}$ realizaram estudo com dados de compras de alimentos da Pesquisa de Orçamentos Familiares (POF) 2008-2009 mostrando a disponibilidade e as fontes de fibras na dieta dos brasileiros. As fontes alimentares com maior contribuição para o total de fibras disponível foram feijões, arroz, pão, vegetais, frutas e farinha de mandioca. Foi observado que a quantidade de fibras disponível para consumo era insuficiente em todas as regiões, áreas e níveis de renda do país, chegando a ser inferior a metade do recomendado pela $\mathrm{OMS}^{7}$.

Não existem dados de representatividade nacional disponíveis descrevendo o consumo efetivo de fibras e suas fontes considerando o grau de processamento alimentar. $\mathrm{O}$ presente estudo objetiva descrever esse consumo, dispondo suas principais fontes alimentares de acordo com características de processamento, assim como avaliar o impacto dos alimentos ultraprocessados no consumo de fibras no Brasil.

\section{Métodos}

O estudo foi realizado a partir de dados coletados pela POF 2008-2009, o inquérito populacional publicado mais recente do país. Esta pesquisa foi realizada pelo Instituto Brasileiro de Geografia e Estatística (IBGE) em uma amostra probabilística dos domicílios brasileiros entre 19 de maio de 2008 e 18 de maio de $2009^{8}$.

A amostra de domicílios foi extraída adotando-se plano de amostragem complexo por conglomerados com sorteio dos setores censitários, em primeiro estágio, e de domicílios, em segundo. Os setores censitários foram selecionados com probabilidade proporcional ao número de domicílios de cada setor, e agrupados previamente para obter estratos de domicílios com suficiente homogeneidade geográfica e socioeconômica. No segundo estágio de seleção foram escolhidos domicílios particulares permanentes, por amostragem aleatória simples, sem reposição, em cada setor censitário. A amostra total de domicílios estudados foi de $55.970^{8}$.

Dados sobre consumo alimentar pessoal foram coletados para todos os moradores com 10 anos ou mais de idade em uma subamostra do total de domicílios, envolvendo 13.569 domicílios $(24,3 \%$ do total) e 34.003 indivíduos 8 .

A entrevista domiciliar, realizada por agentes de pesquisa utilizando microcomputadores portáteis e questionários eletrônicos, compreendeu a coleta de informações socioeconômicas e demográficas de todos os moradores ${ }^{8}$.

O consumo alimentar pessoal foi avaliado por meio de registros alimentares de 24 horas em dois dias não consecutivos. Os indivíduos foram solicitados a registrar todos os alimentos e bebidas consumidos no dia, indicando as quantidades consumidas em medidas caseiras, a forma de preparação e local de consumo. Quantidades consideradas improváveis ou não informadas foram imputadas com base em matriz de similaridades formadas por variáveis consideradas correlacionadas. O sistema de entrada de dados continha cadastro inicial de 1.500 itens de consumo. Estes itens incluíam alimentos, bebidas e preparações que apareceram com maior frequência na base dos dados de aquisição domiciliar da POF 20022003. Itens que não constavam foram incluídos no cadastro final quando referidos pelos entrevistados. Ao final, 1.120 itens de consumo foram efetivamente citados ${ }^{8}$.

As quantidades de cada item de consumo, informadas em medidas caseiras, foram transformadas pelo IBGE em gramas ou mililitros com base na Tabela de Medidas Referidas para os Alimentos Consumidos no Brasil'. As preparações culinárias, ou seja, itens de consumo que possuíam mais de um ingrediente em sua composição, foram desa- 
gregadas utilizando receitas para padronizar as quantidades de seus ingredientes. O primeiro critério adotado para a desagregação foi a orientação dada pelo IBGE quanto à forma de preparo na $\mathrm{Ta}$ bela de Composição Nutricional dos Alimentos Consumidos no Brasil ${ }^{10}$. Na ausência desta informação, utilizamos a Tabela para avaliação de consumo alimentar em medidas caseiras ${ }^{11}$ e o Manual de receitas e medidas caseiras para cálculo de inquéritos alimentares $^{12}$. A partir disso, as quantidades transformadas foram convertidas em energia e fibras alimentares, com base na Tabela de Composição Nutricional dos Alimentos Consumidos no Brasil ${ }^{10}$.

Todos os gêneros alimentícios foram classificados em grupos de acordo com critérios propostos pela classificação $\mathrm{NOVA}^{13}$. O primeiro grupo incluiu alimentos in natura ou minimamente processados, que são partes comestíveis de plantas ou animais, cogumelos e algas, logo após sua separação da natureza, ou quando submetidos a processos como remoção de partes não comestíveis ou não desejadas, desidratação, moagem, fracionamento, torra, pasteurização, congelamento e demais processos que não envolvam a adição de substâncias refinadas. O segundo grupo foi composto pelos ingredientes culinários processados, englobando substâncias extraídas diretamente de alimentos do primeiro grupo ou da natureza, tais como açúcar, sal, óleos e gorduras. O terceiro grupo foi formado pelos alimentos processados, incluindo produtos fabricados com a adição de um ingrediente do segundo grupo a um alimento do primeiro, sendo, em sua maioria, produtos com dois ou três ingredientes. O quarto grupo compreendeu os ultraprocessados, formulações industriais feitas tipicamente com cinco ou mais ingredientes, frequentemente ricos em açúcar, óleos, gorduras e sal, e caracterizados pela presença de corantes, estabilizantes, conservantes e demais aditivos alimentares.

Primeiramente, o total de calorias ingeridas pelos indivíduos foi distribuído segundo os quatro grupos da classificação NOVA ${ }^{13}$ e respectivos subgrupos. A seguir, foi realizado o estudo da contribuição dos grupos para o consumo de fibras no Brasil. Foi calculada a densidade de fibras de cada grupo $(\mathrm{g} / 1.000 \mathrm{kcal})$ e a contribuição percentual relativa de cada um deles para o total de fibras consumidas na dieta.

A análise da associação entre o consumo total de ultraprocessados (\% do total de energia) e a densidade média de fibras da dieta (g/1.000 kcal) foi realizada, empregando-se análises de regressão linear para identificação da direção e do significado estatístico da associação.
Finalmente, avaliou-se a associação entre estratos da população correspondentes a quintis da contribuição dos alimentos ultraprocessados para o total de calorias e a prevalência de consumo inadequado de fibras $(<12,5 \mathrm{~g} / 1.000 \mathrm{kcal})$, empregando-se análises de regressão de Poisson para identificação da direção, magnitude e significado estatístico das associações.

Essas análises foram realizadas sem e com ajuste para potencias confundidores (idade, sexo, anos de estudo, renda, raça/cor, área e região). Foi adotado o nível de significância de 0,05 para todas as análises deste estudo e foram utilizados fatores amostrais de ponderação que permitem a extrapolação dos resultados para a população brasileira. Todas as análises foram efetuadas no software Stata (StataCorp versão 15).

\section{Resultados}

O consumo médio diário de energia dos brasileiros com 10 ou mais anos de idade foi de 1.952,9 kcal (Erro Padrão: 10,4). O consumo médio de fibras foi de 9,88 g/1.000 kcal, sendo que 77\% da população apresentava consumo insuficiente $(<12,5 \mathrm{~g} / 1.000 \mathrm{kcal})^{1}$.

A maior porcentagem da ingestão energética dos brasileiros em 2008-2009 derivou do grupo de alimentos in natura ou minimamente processados (54,1\%), seguida dos ultraprocessados $(18,7 \%)$, ingredientes culinários processados $(16,2 \%)$ e alimentos processados (10,9\%). Dentre os alimentos in natura ou minimamente processados, aqueles com maior contribuição para o total de energia foram carnes e aves (13,5\%), arroz (11,8\%), e feijão e outras leguminosas (10,1\%). Com relação aos ultraprocessados, subgrupos com maior contribuição calórica foram biscoitos, bolos e outros produtos de padaria doces $(4,1 \%)$, pratos prontos para o consumo, congelados ou instantâneos $(3,9 \%)$ e refrigerantes $(1,8 \%)$ (Tabela 1$)$.

O grupo com maior densidade de fibras foi alimentos in natura ou minimamente processados, com 15,4 g/1.000 kcal, destacando vegetais com 63,0 g/1.000 kcal, feijão e outras leguminosas com $38,8 \mathrm{~g} / 1.000 \mathrm{kcal}$, frutas e sucos in natura com 23,7 g/1.000 kcal e raízes e tubérculos com $17,9 \mathrm{~g} / 1.000 \mathrm{kcal}$. Juntos, os alimentos desse gruporepresentaram $80,7 \%$ do total de fibras consumidas pela população, sendo as principais fontes feijão e outras leguminosas $(35,2 \%)$ e arroz $(15,3 \%)$. Somados, esses dois subgrupos corresponderam a mais da metade da ingestão de fibras do brasileiro (Tabela 1). 
Tabela 1. Médias de consumo alimentar relativo de acordo com os grupos e subgrupos da classificação NOVA, densidade de fibras desses alimentos e sua contribuição relativa para o consumo total de fibras por brasileiros com 10 ou mais anos de idade. Brasil, 2008-2009.

\begin{tabular}{|c|c|c|c|}
\hline \multirow[t]{2}{*}{ Grupos de alimentos e itens de consumo } & $\begin{array}{c}\text { Ingestão } \\
\text { Energética } \\
\text { Total } \\
\end{array}$ & $\begin{array}{l}\text { Densidade } \\
\text { de Fibras }\end{array}$ & $\begin{array}{c}\text { Contribuição } \\
\text { de Fibras } \\
\text { Alimentares }\end{array}$ \\
\hline & Média (\%) & $\begin{array}{c}\text { Média } \\
\text { (g/1.000kcal) }\end{array}$ & Média (\%) \\
\hline Alimentos in natura ou minimamente processados & 54,1 & 15,4 & $80,7^{\star}$ \\
\hline Feijão e outras leguminosas & 10,1 & 38,8 & 35,2 \\
\hline Carne e Aves & 13,5 & 0,0 & 0,1 \\
\hline Arroz & 11,8 & 11,7 & 15,3 \\
\hline Massas e outros cereais & 4,5 & 11,2 & 6,1 \\
\hline Raízes e Tubérculos & 3,0 & 17,9 & 5,5 \\
\hline Leite e Iogurte Natural & 1,8 & 0,0 & 0,0 \\
\hline Frutas e Sucos in natura & 5,1 & 23,7 & 11,6 \\
\hline Peixes e Frutos do Mar & 1,3 & 0,0 & 0,0 \\
\hline Ovos & 1,2 & 0,0 & 0,0 \\
\hline Legumes e Verduras & 1,4 & 63,0 & 6,1 \\
\hline Outros alimentos in natura ou minimamente processados ${ }^{\mathrm{a}}$ & 0,4 & 25,2 & 0,8 \\
\hline Ingredientes culinários processados & 16,2 & 0,0 & $0,1^{\star}$ \\
\hline Açúcar de mesa & 7,5 & 0,0 & 0,0 \\
\hline Óleos vegetais & 7,1 & 0,0 & 0,0 \\
\hline Gordura animal & 1,6 & 0,0 & 0,0 \\
\hline Outros ingredientes culinários processados ${ }^{\mathrm{b}}$ & 0,2 & 3,0 & 0,0 \\
\hline Alimentos processados & 10,9 & 6,8 & $8,6^{*}$ \\
\hline Pão Francês & 8,0 & 7,7 & 7,6 \\
\hline Carnes e peixes salgados, defumados ou enlatados & 1,1 & 0,1 & 0,0 \\
\hline Queijos processados & 1,0 & 0,0 & 0,0 \\
\hline $\begin{array}{l}\text { Legumes e outros alimentos em conserva e reservados em } \\
\text { salmoura }\end{array}$ & 0,3 & 25,2 & 0,4 \\
\hline Outros processados ${ }^{\mathrm{c}}$ & 0,6 & 0,0 & 0,0 \\
\hline Alimentos ultraprocessados & 18,7 & 4,9 & $11,2^{*}$ \\
\hline Pães ultraprocessados & 1,4 & 9,3 & 1,5 \\
\hline Refrigerantes & 1,8 & 0,1 & 0,0 \\
\hline Doces & 1,5 & 2,1 & 0,8 \\
\hline Queijos ultraprocessados & 0,1 & 0,0 & 0,0 \\
\hline Carne reconstituída ou produtos à base de peixe & 1,7 & 0,1 & 0,0 \\
\hline Bebidas à base de leite & 1,6 & 12,6 & 2,2 \\
\hline Biscoitos, bolos e outros produtos de padaria doces & 4,1 & 5,4 & 2,9 \\
\hline Pratos prontos para o consumo, congelados ou instantâneos & 3,9 & 5,9 & 3,0 \\
\hline Outras bebidas ultraprocessadas & 0,9 & 4,7 & 0,6 \\
\hline Outros ultraprocessados ${ }^{\mathrm{d}}$ & 1,8 & 11,1 & 0,2 \\
\hline
\end{tabular}

*Todos os valores foram significativamente diferentes entre si. ${ }^{a}$ Nozes e sementes, pimenta, chá e café; ${ }^{\mathrm{b}}$ Amido de milho e vinagre; ${ }^{\mathrm{c} C e r v e j a ~ e ~ v i n h o ; ~}{ }^{\mathrm{d}}$ Molhos, barra de cereal e cereal matinal.

Fonte: Pesquisa de Orçamentos Familiares 2008-2009, IBGE.

O grupo de ingredientes culinários processados não contribuiu com quantidades expressivas de fibras na dieta. Alimentos processados tiveram densidade de fibras de 6,8 g/1.000 kcal e contribuíram com 8,6\% do total de fibras, sendo 7,6\% proveniente do pão francês. A densidade de fibras dos ultraprocessados foi de $4,9 \mathrm{~g} / 1.000 \mathrm{kcal}$ e sua contribuição para o total de fibras consumidas foi de $11,2 \%$, sendo pratos prontos para consumo, congelados ou instantâneos 3,0\% e biscoitos, bolos e outros produtos de padaria doces 2,9\% (Tabela 1). 
A análise de associação entre o consumo de ultraprocessados (\% de energia da dieta) e a densidade de fibras $(\mathrm{g} / 1.000 \mathrm{kcal})$ demonstrou que o aumento de $10 \%$ na ingestão de ultraprocessados determinou uma diminuição do consumo médio de fibras de 0,70 g/1.000 kcal (IC95\% -0,74; 0,67) (Figura 1).

Observou-se aumento da prevalência do consumo insuficiente de fibras com o aumento do quintil de consumo de ultraprocessados. No primeiro quintil, 63,8\% dos indivíduos apresentaram consumo insuficiente de fibras; no quinto quintil esse número alcançou 93,1\%. Indivíduos no maior quintil de consumo de ultraprocessados possuíram 50\% mais chance de terem ingestão insuficiente de fibras do que aqueles no menor quintil ( $\mathrm{RP}=1,5$; IC95\% 1,4-1,5). O ajuste para variáveis sociodemográficas não alterou substancialmente os resultados (Tabela 2).

\section{Discussão}

Os resultados deste estudo demonstram que a maior parte dos brasileiros tem um consumo de fibras insuficiente e confirmaram o perfil nutricional desfavorável dos alimentos ultraprocessados em relação à ingestão desse nutriente, docu- mentando seu impacto negativo na qualidade da alimentação da população. Quanto maior o consumo de ultraprocessados, menor a ingestão de fibras e maior a prevalência de consumo inferior ao recomendado pela OMS.

A ingestão de fibras está majoritariamente associada a alimentos in natura ou minimamente processados; a densidade de fibras desse grupo superou em três vezes a dos ultraprocessados. A maior contribuição de fibras para o brasileiro derivou dos subgrupos arroz e feijões, documentando a importância nutricional dessa combinação alimentar típica da cultura do país.

Há uma tendência observada no Brasil em substituir refeições tradicionais baseadas em alimentos in natura ou minimamente processados por alimentos ultraprocessados. Entre 2002 e 2009, o consumo de ultraprocessados teve aumento significativo de $5 \%$ no Brasil, enquanto o consumo de arroz e feijão passou de $22,8 \%$ para $20,6 \%$, caindo $2,2 \%{ }^{14}$.

É observado, em todo o mundo, o avanço do consumo de alimentos ultraprocessados em substituição à preparações culinárias tradicionais baseadas em alimentos in natura e minimamente processados ${ }^{13}$. Em países de média renda, as vendas de ultraprocessados crescem até $10 \%$ ao ano ${ }^{15}$ e em países de alta renda, como Estados Unidos,

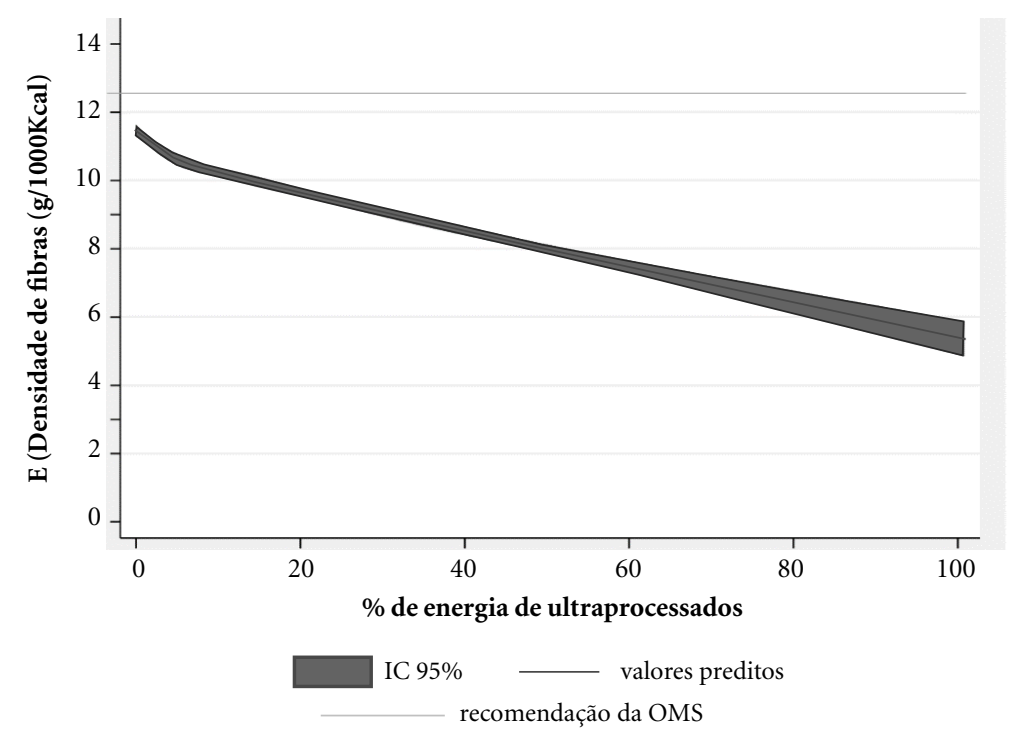

Figura 1. Associação entre o consumo de ultraprocessados (\% do total de energia) e a ingestão de fibras alimentares (g/1.000kcal) na população brasileira com 10 ou mais anos de idade. Brasil, 2008-2009. 
Tabela 2. Distribuição (\%) da ingestão de fibras alimentares em estratos da população brasileira com 10 ou mais anos de idade correspondentes a quintis do consumo de alimentos ultraprocessados. Brasil, 2008-2009.

\begin{tabular}{lcccccc}
\hline \multicolumn{2}{c}{$\begin{array}{c}\text { Contribuição de } \\
\text { ultraprocessados na dieta } \\
\text { do total de energia) }\end{array}$} & \multicolumn{6}{c}{ Participantes com consumo de fibras inferior a 12,5g/1000kcal } \\
\hline Quintis & Média & $\begin{array}{c}\text { Prevalência } \\
(\%)\end{array}$ & RP & IC95\% & RP ajustada $^{\text {a }}$ & IC95\% \\
\hline $1^{\text {o }}$ & 0,1 & 63,8 & - & - & - & - \\
$2^{\text {o }}$ & 5,9 & 71,4 & 1,1 & 1,$1 ; 1,2$ & 1,1 & 1,$1 ; 1,1$ \\
$3^{\text {o }}$ & 13,8 & 75,9 & 1,2 & 1,$1 ; 1,2$ & 1,2 & 1,$1 ; 1,2$ \\
$4^{\text {o }}$ & 25,4 & 82,7 & 1,3 & 1,$3 ; 1,3$ & 1,3 & 1,$2 ; 1,3$ \\
$5^{\text {o }}$ & $48,7^{\text {b }}$ & 93,1 & $1,5^{\text {b }}$ & 1,$4 ; 1,5$ & $1,4^{\text {b }}$ & 1,$4 ; 1,4$ \\
\hline
\end{tabular}

RP: Razão de prevalência; IC: Intervalo de Confiança. ${ }^{~}$ Razão de Prevalência ajustada para idade, sexo, anos de estudo, renda, raça/cor, área e região; ${ }^{b} \mathrm{p}<0,05$ para tendência linear da variação do indicador conforme quintis do consumo de alimentos ultraprocessados.

Fonte: Pesquisa de Orçamentos Familiares 2008-2009, IBGE.

Canadá, Reino Unido e Austrália, seu consumo representa pelo menos metade da energia total da dieta consumida ${ }^{16-19}$.

Alimentos ultraprocessados sofrem diversas etapas de manipulação industrial para se tornarem duráveis e hiperpalatáveis, usualmente implicando na destruição da integridade da matriz alimentar acompanhada da retirada das fibras $^{4,20,21}$. Sua reformulação para adição de fibras não parece vantajosa, uma vez que, salvo casos de deficiência clínica, nutrientes isolados não demonstram efeitos significativos para o benefício da saúde de populações ${ }^{20,22}$. Ademais, a FAO e a OMS recomendam que a ingestão adequada de fibras deve ser alcançada por meio de fontes alimentares naturais ${ }^{1,23}$. Autores têm destacado os potenciais riscos da reformulação de ultraprocessados para promoção da alimentação saudável ${ }^{22}$.

O presente estudo demonstrou que o consumo de ultraprocessados impacta negativamente na ingestão de fibras, que possuem efeito protetor em diversas doenças. Entretanto, suas consequências adversas na saúde vão além. Estudos evidenciaram associação entre o consumo de alimentos ultraprocessados e aumento do IMC, maior risco de desenvolvimento de obesidade, doença cardiovascular, hipertensão, acidente vascular cerebral, síndrome metabólica e cânceres no geral ${ }^{24-28}$.

Análises realizadas com dados de representatividade nacional dos Estados Unidos e do Reino Unido, onde os padrões alimentares se diferenciam significativamente do Brasil e contemplam maior consumo de ultraprocessados, também observaram uma redução no consumo de fibras associada ao aumento da ingestão de ultraprocessados $^{29,30}$.

Convém destacar que, mesmo no quintil com menor consumo de ultraprocessados, a prevalência de ingestão inadequada de fibras ainda é alta. Isso se deve, possivelmente, à baixa diversidade da fração não ultraprocessada da alimentação do brasileiro, somada ao consumo elevado de alimentos de origem animal e ao baixo consumo de legumes e verduras.

A adequação do consumo de fibras deve ser almejada, portanto, minimizando a ingestão de ultraprocessados e enfatizando o consumo de alimentos in natura ou minimamente processados em grande variedade e predominantemente de origem vegetal ${ }^{4}$.

Políticas públicas, programas e estratégias que incentivem e viabilizem o acesso a alimentos in natura e minimamente processados devem ser fomentados, assim como o desenvolvimento de ações que resgatem e promovam a alimentação saudável tradicional. O Programa Bolsa Família, criado em 2003, é um exemplo de política pública que facilitou o acesso de famílias de baixa renda a alimentos in natura e minimamente processados. Como aponta estudo, famílias beneficiadas pelo programa tiveram maior consumo de alimentos desse grupo, incluindo hortaliças, quando comparadas a famílias sem o benefício ${ }^{31}$. Outro exemplo é o Programa Nacional de Alimentação Escolar (PNAE), em vigor no Brasil, que determina a incorporação obrigatória de frutas e hortaliças na alimentação escolar da rede pública de ensi$n^{32}$, além de estar associado a um menor consumo de ultraprocessados pelas crianças ${ }^{33}$. 
Os Estados Unidos possuem políticas de zoneamento para garantir e disseminar a presença de locais que comercializem alimentos saudáveis, assim como políticas de incentivo ao acesso a alimentos provenientes de pequenos produtores. Tais intervenções têm como resultado o aumento da ingestão de frutas e vegetais, cujo consumo está inversamente associado com a ingestão de ultraprocessados $^{34,35}$. Todas as ações citadas, ao impulsionarem o consumo de alimentos in natura e minimamente processados, contribuem para o aumento da ingestão de fibras alimentares na dieta.

Junto às ações de promoção da alimentação adequada e saudável, é relevante a adoção de medidas regulatórias para as indústrias de ultraprocessados. Dentre as medidas possíveis, inclui-se as taxações e intervenções no preço dos produtos ultraprocessados, a regulação de propagandas e promoções comerciais para o público infantojuvenil e a adequação da rotulagem nutricional que facilite sua compreensão e possibilite a população fazer escolhas de alimentos mais saudáveis para consumo ${ }^{36}$. Essas medidas são de reconhecida importância e necessitam ser adotadas com urgência. Revisão sistemática de alcance global mostrou que taxações de bebidas açucaradas e de outros ultraprocessados foram eficazes em reduzir sua aquisição, assim como seu consumo ${ }^{37}$.

Dentre os pontos fortes deste estudo, destacam-se o caráter rigorosamente probabilístico da amostra e a representatividade nacional, assegu- rada com a análise de mais de 30 mil pessoas residentes nas áreas urbanas e rurais das várias regiões do país; e o emprego da classificação NOVA, adotada oficialmente pelo Ministério da Saúde no Guia Alimentar para a População Brasileira ${ }^{4}$.

As limitações se relacionam com vieses potenciais inerentes ao uso de registros alimentares, como subestimação do consumo, modificação da ingestão habitual nos dias do estudo, diferenças entre receitas culinárias reais e receitas padronizadas e diferenças entre a composição nutricional real dos alimentos e a composição indicada pela tabela de composição nutricional utilizada. Para minimizar parte desses vieses, o instrumento de coleta foi pré-testado e validado, procedimentos de controle de qualidade foram realizados durante a coleta de dados e registros inconsistentes foram excluídos e substituídos com valores imputados $^{8}$. Esta investigação determina resultados aplicados apenas à população com 10 ou mais anos de idade.

Esse foi o primeiro estudo com representatividade nacional a descrever o consumo de fibras e suas fontes de acordo com o grau de processamento alimentar. $\mathrm{O}$ consumo de ultraprocessados impacta negativamente na ingestão de fibras; evitar o consumo de ultraprocessados aumentando a quantidade e variedade de alimentos in natura e minimamente processados tem grande potencial para aumentar substancialmente a quantidade de fibras na dieta.

\section{Colaboradores}

GL da Cruz contribuição na análise, interpretação dos dados e redação do manuscrito. PP Machado contribuiu na análise, interpretação dos dados e na revisão do manuscrito. GC Andrade contribuiu na análise, interpretação dos dados e na revisão do manuscrito. MLC Louzada contribui na concepção e desenho, análise e interpretação dos dados e revisão do manuscrito. Todas as autoras aprovaram a versão final do artigo. 


\section{Referências}

1. World Health Organization (WHO). Diet, nutrition and the prevention of chronic diseases: report of a Joint WHO/FAO Expert Consultation. Geneva: WHO; 2003.

2. Institute of Medicine, Food and Nutrition Board. Dietary Reference Intakes: Energy, Carbohydrates, Fiber, Fat, Fatty Acids, Cholesterol, Protein and Amino Acids. National Academies Press: Washington, DC; 2002.

3. Slavin JL. Position of the American Dietetic Association: Health Implications of Dietary Fiber. J Am Diet Assoc 2008; 108(10):1716-1731.

4. Brasil. Ministério da Saúde (MS). Guia alimentar para a população brasileira. 2a ed. Brasília: MS; 2014.

5. U.S. Department of Agriculture and U.S. Department of Health and Human Services. Dietary Guidelines for Americans, 2010. 7a ed. Washington, DC: U.S. Government Printing Office; 2010.

6. Public Health England. National Diet and Nutrition Survey. Results from Years 7-8 (Combined) of the Rolling Programme (2014/15 to 2015/16). Londres: Public Health England; 2018.

7. Sardinha AN, Canella DS, Martins APB, Claro RM, Levy RB. Dietary sources of fiber intake in Brazil. Appetite 2014; 79:134-138.

8. Instituto Brasileiro de Geografia e Estatística (IBGE). Pesquisa de orçamentos familiares 2008-2009: análise do consumo alimentar pessoal no Brasil. Rio de Janeiro: IBGE; 2011.

9. Instituto Brasileiro de Geografia e Estatística (IBGE). Pesquisa de orçamentos familiares 2008-2009: tabela de medidas referidas para os alimentos consumidos no Brasil. Rio de Janeiro: IBGE; 2011.

10. Instituto Brasileiro de Geografia e Estatística (IBGE). Pesquisa de orçamentos familiares 2008-2009: tabelas de composição nutricional dos alimentos consumidos no Brasil. Rio de Janeiro: IBGE; 2011.

11. Pinheiro ABV, Lacerda EMA, Benzecry EH, Gomes MCS. Tabela para avaliação de consumo alimentar em medidas caseiras. $5^{\mathrm{a}}$ ed. São Paulo: Atheneu; 2008.

12. Fisberg RM, Villar BS. Manual de receitas e medidas caseiras para cálculo de inquéritos alimentares: manual elaborado para auxiliar o processamento de dados de inquéritos alimentares. São Paulo: Signus; 2002.

13. Monteiro CA, Cannon G, Levy RB, Moubarac J-C, Jaime P, Martins AP, Canella D, Louzada M, Parra D, Ricardo C, Calixto G, Machado P, Martins C, Martinez E, Baraldi L, Garzillo J, Sattamini I. NOVA. A estrela brilha. World Nutr J 2016; 7(1-3):28-40.

14. Martins APB, Levy RB, Claro RM, Moubarac JC, Monteiro CA. Increased contribution of ultra-processed food products in the brazilian diet (1987-2009). Rev Saude Publica 2013; 47(4):656-665.

15. Monteiro CA, Moubarac J-C, Cannon G, Ng SW, Popkin B. Ultra-processed products are becoming dominant in the global food system. Obes Rev 2013; 14(2):21-28.

16. Baraldi LG, Steele EM, Canella DS, Monteiro CA. Consumption of ultra-processed foods and associated sociodemographic factors in the USA between 2007 and 2012: evidence from a nationally representative crosssectional study. BMJ Open 2018; 8(3):e020574.
17. Moubarac JC, Batal M, Louzada ML, Steele EM, Monteiro CA. Consumption of ultra-processed foods predicts diet quality in Canada. Appetite 2017; 108:512520.

18. Rauber F, Louzada MLC, Steele EM, Millett C, Monteiro CA, Levy RB. Ultraprocessed food consumption and chronic non-communicable diseases-related dietary nutrient profile in the UK (2008-2014). Nutrients 2018; 10(5):E587.

19. Machado PP, Steele EM, Levy RB, Sui Z, Rangan A, Woods J, Gill T, Scrinis G, Monteiro CA. Ultra-processed foods and recommended intake levels of nutrients linked to noncommunicable diseases in Australia: evidence from a nationally representative crosssectional study. BMJ Open 2019; 9(8):e029544.

20. Jacobs DR, Tapsell LC. Food synergy: the key to a healthy diet. Proc Nutr Soc 2013; 72(2):200-206.

21. Tapsell LC, Neale EP, Satija A, Hu FB. Foods, nutrients and dietary patterns: interconnections and implications for dietary guidelines. Adv Nutr 2016; 7(3):445454.

22. Scrinis G, Monteiro CA. Ultra-processed foods and the limits of product reformulation. Public Health Nutr 2018; 21(1):247-252.

23. World Health Organization (WHO). Food and Agriculture Organization of the United Nations (FAO). Carbohydrates in human nutrition. Report of a Joint FAO/WHO expert consultation. FAO Food Nutr Pap 1998; 66:1-140.

24. Louzada MLC, Baraldi LG, Steele EM, Martins APB, Canella DS, Moubarac J-C, Levy RB, Cannon G, Afshin A, Imamura F, Mozaffarian D, Monteiro CA. Consumption of ultra-processed foods and obesity in Brazilian adolescents and adults. Prev Med 2015; 81:9-15.

25. Mendonça RD, Lopes ACS, Pimenta AM, Gea A, Martinez-Gonzalez MA, Bes-Rastrollo M. Ultra-processed food consumption and the incidence of hypertension in a mediterranean cohort: the seguimiento Universidad de Navarra Project. Am J Hypertens 2017; 30(4):358-366.

26. Steele EM, Juul F, Neri D, Rauber F, Monteiro CA. Dietary share of ultra-processed foods and metabolic syndrome in the US adult population. Prev Med 2019; 125:40-48.

27. Fiolet T, Srour B, Sellem L, Kesse-Guyot E, Allès B, Méjean C, Deschasaux M, Fassier P, Latino-Martel P, Beslay M, Hercberg S, Lavalette C, Monteiro CA, Julia C, Touvier M. Consumption of ultra-processed foods and cancer risk: results from NutriNetSanté prospective cohort. BMJ 2018; 360:k322.

28. Srour B, Fezeu LK, Kesse-Guyot E, Allès B, Méjean C, Andrianasolo RM, Chazelas E, Deschasaux M, Hercberg S, Galan P, Monteiro CA, Julia C, Touvier M. Ultra-processed food intake and risk of cardiovascular disease: prospective cohort study (NutriNet-Santé). BMJ 2019; 365:11451.

29. Rauber F, Louzada MLC, Steele EM, Millett C, Monteiro CA, Levy RB. Ultra-Processed Food Consumption and Chronic Non-Communicable Diseases-Related Dietary Nutrient Profile in the UK (2008- 2014). Nutrients 2018; 10(5):587. 
30. Steele EM, Popkin BM, Swinburn B, Monteiro CA. The share of ultra-processed foods and the overall nutritional quality of diets in the US: evidence from a nationally representative cross-sectional study. Popul Health Metr 2017; 15(1):6.

31. Martins APB, Monteiro CA. Impact of the Bolsa Família program on food availability of low-income Brazilian families: a quasi experimental study. $B M C$ Public Health 2016; 16(1):827.

32. Fundo Nacional de Desenvolvimento da Educação (FNDE). Resolução CD/FNDE no 26, de 17 de junho de 2013. Dispõe sobre o atendimento da alimentação escolar aos alunos da educação básica no âmbito do Programa Nacional de Alimentação Escolar - PNAE. Diário Oficial da União 2013; 17 jun..

33. Noll PR, Noll M, Abreu LC, Baracat EC, Silveira EA, Sorpreso ICE. Ultra-processed food consumption by Brazilian adolescents in cafeterias and school meals. Sci Rep 2019; 9(1):7162.

34. Jilcott Pitts SB, Acheson MLM, Ward RK, Wu Q, McGuirt JT, Bullock SL, Lancaster MF, Raines J, Ammerman AS. Disparities in healthy food zoning, farmers' market availability, and fruit and vegetable consumption among North Carolina residents. Arch Public Health 2015; 73(1):35.

35. Costa JC, Canella DS, Martins APB, Levy RB, Andrade GC, Louzada MLC. Consumo de frutas e associação com a ingestão de alimentos ultraprocessados no Brasil em 2008-2009. Cien Saude Colet 2021; 26(4):12331244.

36. Corvalán C, Reyes M, Garmendia ML, Uauy R. Structural responses to the obesity and non-communicable diseases epidemic: the Chilean Law of Food Labeling and Advertising. Obes Rev 2013; 14(Supl. 2):79-87.

37. Teng AM, Jones AC, Mizdrak A, Signal L, Genç M, Wilson N. Impact of sugar-sweetened beverage taxes on purchases and dietary intake: Systematic review and meta-analysis. Obes Rev 2019; 20(9):1187-1204.

Artigo apresentado em 19/06/2019

Aprovado em 29/06/2020

Versão final apresentada em 02/07/2020

Editores-chefes: Romeu Gomes, Antônio Augusto Moura da Silva 
